\title{
Comparison of Clinical and Radiological Results of Posterolateral Fusion and Posterior Lumbar Interbody Fusion in the Treatment of L4 Degenerative Lumbar Spondylolisthesis
}

\author{
Shugo Kuraishi ${ }^{1}$, Jun Takahashi ${ }^{1}$, Keijiro Mukaiyama ${ }^{2}$, Masayuki Shimizu ${ }^{1}$, \\ Shota Ikegami ${ }^{1}$, Toshimasa Futatsugi ${ }^{1}$, Hiroki Hirabayashi ${ }^{3}$, Nobuhide Ogihara ${ }^{4}$, \\ Hiroyuki Hashidate ${ }^{5}$, Yutaka Tateiwa ${ }^{6}$, Hisatoshi Kinoshita ${ }^{6}$, Hiroyuki Kato ${ }^{1}$ \\ ${ }^{1}$ Department of Orthopaedic Surgery, Shinshu University School of Medicine, Matsumoto, Japan \\ ${ }^{2}$ Department of Orthopaedic Surgery, Azumi General Hospital, Nagano,Japan \\ ${ }^{3}$ Department of Orthopaedic Surgery, Marunouchi Hospital, Matsumoto, Japan \\ ${ }^{4}$ Department of Orthopaedic Surgery, Ina Central Hospital, Ina, Japan \\ ${ }^{5}$ Department of Orthopaedic Surgery, Shinonoi General Hospital, Nagano, Japan \\ ${ }^{6}$ Nagano Prefectural General Rehabilitation Center, Nagano, Japan
}

Study Design: Multicenter analysis of two groups of patients surgically treated for degenerative $L 4$ unstable spondylolisthesis.

Purpose: To compare the clinical and radiographic outcomes of posterolateral fusion (PLF) and posterior lumbar interbody fusion (PLIF) for degenerative $L 4$ unstable spondylolisthesis.

Overview of Literature: Surgery for lumbar degenerative spondylolisthesis is widely performed. However, few reports have compared the outcome of PLF to that of PLIF for degenerative L4 unstable spondylolisthesis.

Methods: Patients with $L 4$ unstable spondylolisthesis with Meyerding grade II or more, slip of $>10^{\circ}$ or $>4 \mathrm{~mm}$ upon maximum flexion and extension bending, and posterior opening of $>5$ degree upon flexion bending were studied. Patients were treated from January 2008 to January 2010. Patients who underwent PLF $(n=12)$ and PLIF $(n=19)$ were followed-up for $>2$ years. Radiographic findings and clinical outcomes evaluated by the Japanese Orthopaedic Association (JOA) score were compared between the two groups. Radiographic evaluation included slip angle, translation, slip angle and translation during maximum flexion and extension bending, intervertebral disc height, lumbar lordotic angle, and fusion rate.

Results: JOA scores of the PLF group before surgery and at final follow-up were 12.3 \pm 4.8 and $24.1 \pm 3.7$, respectively; those of the PLIF group were 14.7 \pm 4.8 and $24.2 \pm 7.8$, respectively, with no significant difference between the two groups. Correction of slip estimated from postoperative slip angle, translation, and maintenance of intervertebral disc height in the PLIF group was significantly $(p<0.05)$ better than those in the PLF group. However, there was no significant difference in lumbar lordotic angle, slip angle and translation angle upon maximum flexion, or extension bending. Fusion rates of the PLIF and PLF groups had no significant difference.

Conclusions: The L4-L5 level posterior instrumented fusion for unstable spondylolisthesis using both PLF and PLIF could ameliorate clinical symptoms when local stability is achieved.

Keywords: Degenerative spondylolisthesis; Instability; Posterolateral fusion; Posterior lumbar interbody fusion

\footnotetext{
Received Nov 26, 2014; Revised May 27, 2015; Accepted May 28, 2015

Corresponding author: Jun Takahashi

Department of Orthopaedic Surgery, Shinshu University School of Medicine,

3-1-1 Asahi, Matsumoto 390-8621, Japan

Tel: +81-263-37-2659, Fax: +81-263-35-8844, E-mail: jtaka@shinshu-u.ac.jp
} 


\section{Introduction}

Surgical treatment of degenerative lumbar spondylolisthesis is controversial. Such procedures include decompression alone or with fusion (in-situ fusion or fusion with correction). Simple posterior decompression in patients without lumbar instability is reported to achieve good short-term results. However, symptoms can recur within a few years [1,2]. Thus, current consensus is that fusion is necessary for degenerative lumbar spondylolisthesis.

Development of pedicle screws has improved clinical results $[3,4]$. Posterior lumbar interbody fusion (PLIF) that employs interbody fusion and posterior lateral fusion (PLF) that does not employ interbody fusion are widely performed. However, which one is an optimal surgical approach remains controversial.

Anterior column support by PLIF is expected to achieve better clinical results than PLF as it can achieve lumbar lordosis recovery, slip correction, and indirect foraminal decompression [5]. In addition, bone graft after debridement of degenerated lumbar discs has a positive effect on bony fusion [6,7]. Given these advantages, numerous surgeons have performed PLIF for the treatment of degenerative spondylolisthesis [8-13].

Many studies have compared these two approaches. However, few reports have focused on a single vertebral level. Indications of these approaches remain unclear. Therefore, the objective of this study was to retrospectively compare the clinical outcomes of PLF and PLIF for degenerative spondylolisthesis of the fourth lumbar vertebra showing predicted instability on plain radiographic evaluation.

\section{Materials and Methods}

\section{Patients}

This study was approved by the ethical committees of two institutions. In these two facilities, PLF and PLIF were performed for 24 and 46 patients, respectively, with degenerative lumbar spondylolisthesis in the fourth vertebra from January 2008 to January 2010. All PLF procedures were performed in Shinshu University Hospital because Nagano General Rehabilitation Center indicated PLIF for all degenerative spondylolisthesis cases during that period. Subjects who underwent single vertebral level (L4-L5) decompression for unstable degenerative spondylolisthe- sis of the fourth lumbar vertebra with the following conditions and were followed up for $\geq 2$ years were retrospectively studied: Meyerding grade II or more, slip of $\geq 10^{\circ}$ or $\geq 4 \mathrm{~mm}$ in the maximum flexion and extension, and posterior opening of $\geq 5^{\circ}$ in the maximum flexion. Twelve subjects (11 men, 1 women; mean age, $69.5 \pm 7.7$ years) underwent PLF with a mean follow-up period of $2.8 \pm 0.9$ years. A total of 19 subjects ( 7 men, 12 women; mean age, 69.4 \pm 6.0 years) underwent PLIF with a mean followup period of $2.6 \pm 0.7$ years. The clinical outcomes were evaluated by performing radiographic imaging. Japanese Orthopaedic Association (JOA) scores were compared between the PLF and PLIF groups.

\section{Surgical methods}

For the PLF procedure, decortications of the transverse process and local bone graft of the inter-transverse process was performed after posterior decompression and instrumentation with a pedicle screw. Hydroxyapatite granules or beta-tricalcium phosphate granules were added if the harvested local bone was insufficient. For the PLIF procedure, posterior decompression by bilateral total facetectomy and pedicle screw instrumentation was performed. Correction was achieved by using screw depth gap. Disc space curettage procedure was performed by distraction between screws and cutting the vertebral disc to the cartilage endplate. Fixation was performed using local bone grafting, placement of a local bone-filled cage, and addition of a compression force. Cage selection was made according to surgeon's preference.

\section{Outcome measurements}

1) Clinical evaluation

JOA score and JOA subscores for back pain and walking ability were measured preoperatively, 1, 3, 6 months, 1 year postoperatively and at the final follow up. Improvement rates were calculated by using Hirabayashi's method [14] and JOA scores before surgery, 1, 3, 6 months, 1 year postoperatively and at the final follow-up using the following equation: (postoperative score-preoperative score)/(29 points-preoperative score $) \times 100 \%$. Length of hospital stay, surgical time, intraoperative blood loss, and postoperative blood loss until drainage removal were measured. Postoperative complications were also studied. 


\section{2) Radiographic evaluation}

Slip length and angle, slip ratio, disc height, and lumbar lordosis (L1-S1) were assessed preoperatively, 1 week, 3 months, 6 months, 1 year postoperatively and at the final follow-up. Slip length and angle were measured by performing radiographic kymography on maximum flexion and extension preoperatively, 3, 6 months, 1 year postoperatively and at the final follow-up.

To control bias caused by the magnification factor on radiographic imaging, slip ratio (\% of slip) was measured. Length of the upper endplate of the L5 vertebra was defined as $100 \%$. Disc height was measured as follows: a vertical line was made from the midpoint of the line between the anterosuperior margin of the L5 vertebral body and posteroinferior margin of the L4 vertebral body to the L5 upper endplate. The distance from the endplate to L4-L5 was measured. To correct bias due to radiographic image magnification, the percentage of disc height was calculated as the ratio to $\mathrm{L} 4$ posterior vertebral height $(\mathrm{H})$ as follows: disc height $\%=$ postoperative disc height $(\mathrm{h}) /$ posterior wall height of the L4 vertebral body $(\mathrm{H})$. Lumbar lordosis was evaluated by the angle between the superior edge of the vertebral body of L1 and S1 (Fig. 1).

Bony union was evaluated at the final follow-up. Fusion was defined as follows: In PLF, bilateral or unilateral fusion mass was observed between the transverse processes on plain radiograph or CT and radiographic kymography of the maximum flexion and extension revealed a slip angle difference of $<4^{\circ}$; In PLIF, bone fusion seen on the radiographs was classified into 4 grades [15]. Grade 1 was defined when a complete fusion achieved with bone bridge formation between the upper and lower vertebral bodies was observed. Grade 2 was defined when bone bridge was not formed but there was no translucency observed around the cages with thick fusion mass formation. Grade 3 was defined when fusion was not achieved with translucency seen around the cages. Grade 4 was defined when cage sunk into the vertebral body or there was bone resorption around cages indicating pseudarthrosis. We aslso measured the standing flexion-extension angle to evaluate mobility. Result was labeled as "achieved fusion" when it was grade 1 or grade 2 with a flexionextension angle of less than $4^{\circ}[15,16]$. It was labeled as "nonfusion" when it was rated grade 3 with flexionextension angle measuring $5^{\circ}$ or more. It was labeled as “pseudarthrosis” when it was rated grade 4.

\section{Statistics}

Student's $t$-tests were used to compare clinical and radiologic measurements. Fisher's exact test was used to compare the rates of intertransverse fusion. All analyses were performed by using the SPSS ver. 10.0 (IBM Co., Endicott, NY, USA). Statistical significance was considered when $p$ value was less than 0.05 .

\section{Results}

\section{Clinical results}

The age and sex distributions were in the PLIF group were similar to those in the PLF group (Table 1). The JOA scores of the PLF group preoperatively, 1, 3, 6 months, 1 year postoperatively and at the final follow-up were $12.1 \pm 5.0,23.4 \pm 2.3,21.8 \pm 4.1,22.1 \pm 3.5,24.2 \pm 3.35$, and $23.9 \pm 3.1$, respectively. Those of the PLIF group were
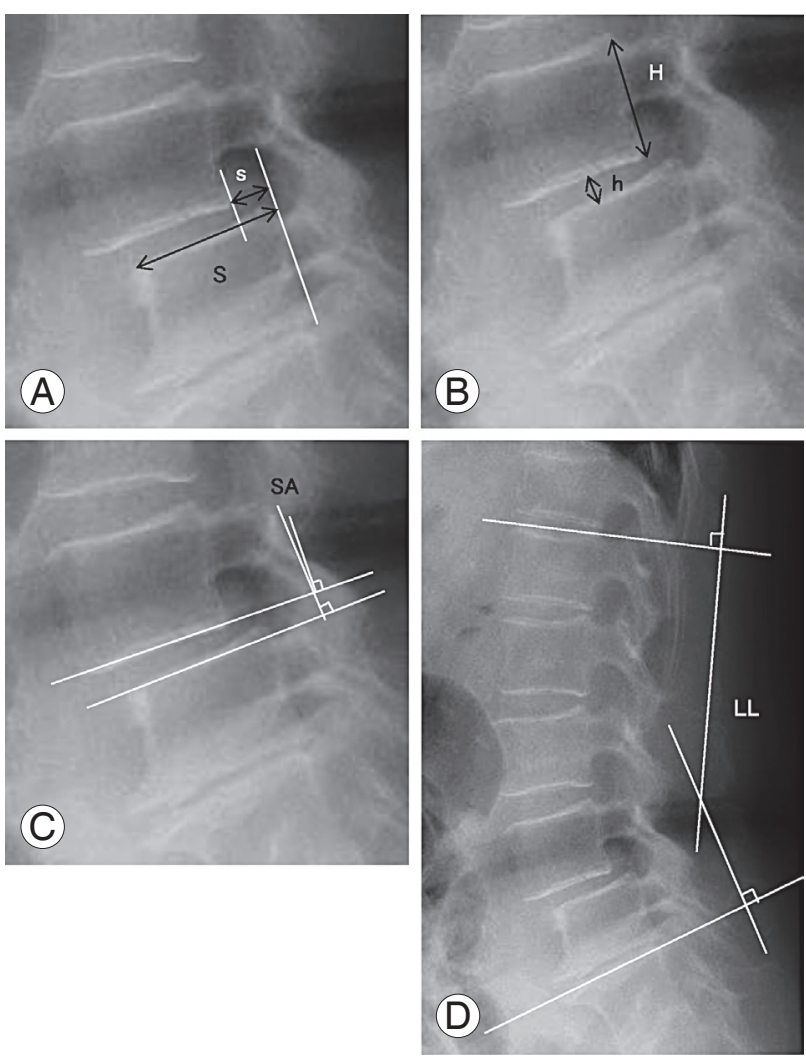

Fig. 1. Radiologic measurements of (A) percentage slip, (B) disc height, (C) slip angle, and (D) lumbar lordosis. The SA, degree of slip (\% of slip), and disc height $(\mathrm{h} / \mathrm{H})$ were measured for the sagittal profile. $\mathrm{LL}$ was measured for alignment. S, slippage; $h$, disc height; $H$, posterior wall height of the proximal vertebral body; $S A$, slip angle; $L L$, lumbar lordosis. 
Table 1. Demographics for each type of treatment

\begin{tabular}{lccc} 
Variable & PLF $(\mathrm{n}=12)$ & PLIF $(\mathrm{n}=19)$ & $p$-value \\
Age (yr) & $69.5 \pm 6.4$ & $69.4 \pm 7.7$ & 0.76 \\
\% (no.) of women & 81.8 & 63.1 & 0.24 \\
\hline Length (cm) & $151.8 \pm 10.1$ & $156.9 \pm 8.7$ & 0.2 \\
Weight (kg) & $57.0 \pm 9.4$ & $57.2 \pm 9.9$ & 0.55 \\
\hline Follow-up period (yr) & 2.7 & 2.6 & 0.58 \\
\hline
\end{tabular}

PLF, posterior lumbar fusion; PLIF, posterior lumbar interbody fusion.

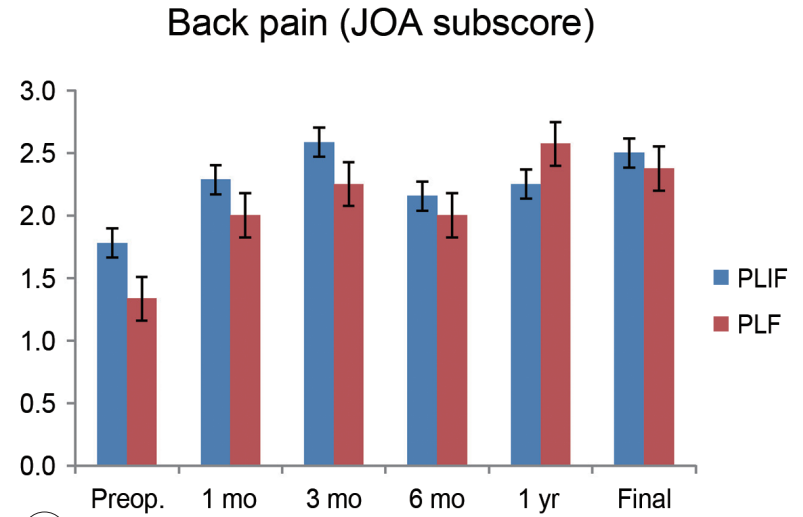

(A)

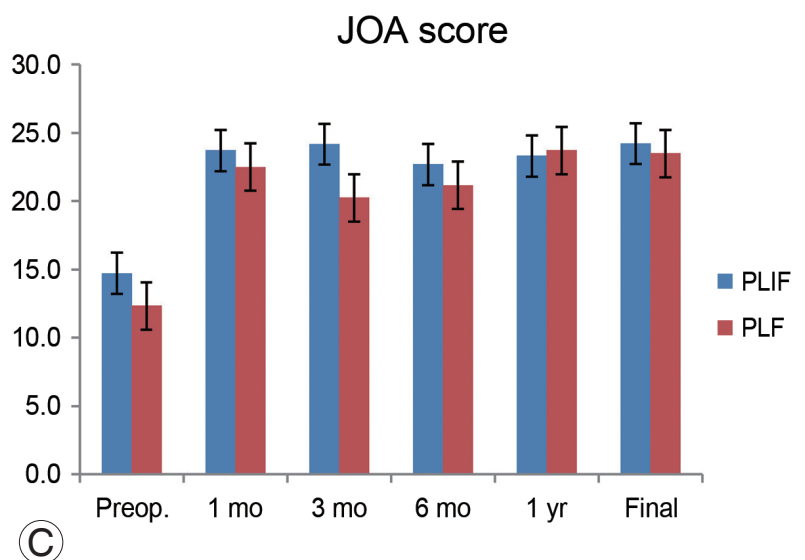

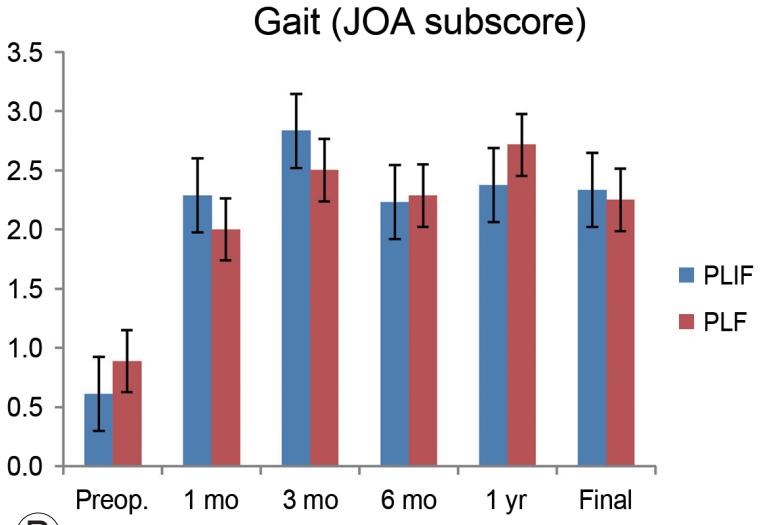

(B)

The improvement rate by the Hirabayashi method

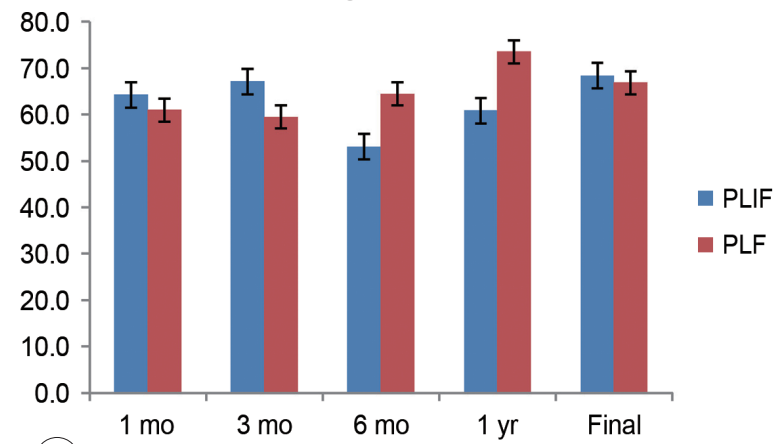

(D)

Fig. 2. (A-D) Clinical data. There was no significant difference in total JOA score, JOA subscore of back pain, or walking ability between PLF and PLIF groups at each follow-up time point. JOA, Japanese Orthopaedic Association; Preop., preoperative; PLIF, posterior lumbar interbody fusion; PLF, posterior lumbar fusion.

$14.8 \pm 5.0,23.7 \pm 1.6,24.2 \pm 2.3,22.7 \pm 4.3,23.4 \pm 5.6$, and $24.3 \pm 5.3$, respectively. There was no significant difference between the two groups. JOA walking function subscores of the PLF group preoperatively, 1, 3, 6 months, 1 year postoperatively and at the final follow-up were $0.8 \pm 0.6$, $2.2 \pm 0.8,2.6 \pm 0.5,2.4 \pm 0.7,2.7 \pm 0.4$, and $2.4 \pm 0.7$, respectively. Those of the PLIF group were $0.6 \pm 0.8,2.1 \pm 1.0$, $2.8 \pm 0.4,2.2 \pm 1.0,2.4 \pm 0.9$, and $2.4 \pm 1.0$, respectively. There was no significant difference between the two groups. The JOA back pain subscores of the PLF group preoperatively, 1, 3, 6 months, 1 year postoperatively, and at final follow-up were $1.3 \pm 0.8,2.0 \pm 0.8,2.4 \pm 0.5,2.0 \pm 0.5,2.5 \pm 0.5$, and 2.3 \pm 0.7 , respectively. Those of the PLIF group were $1.8 \pm 0.8,2.3 \pm 0.7,2.5 \pm 0.5,2.2 \pm 0.8,2.2 \pm 0.9$, and $2.5 \pm 0.6$, respectively. There was no significant difference between the two groups (Fig. 2). 
Table 2. Clinical data

\begin{tabular}{lccc} 
Variable & PLF $(n=12)$ & PLIF (n=19) & $p$-value \\
The number of days in the hospital & $24.5 \pm 8.7$ & $27.4 \pm 12.2$ & 0.52 \\
\hline Operating time (min) & $196.5 \pm 9.5$ & $108.6 \pm 19.5$ & $<0.01$ \\
\hline The amount of blood lost during operation $(\mathrm{mL})$ & $325.5 \pm 191.6$ & $177.3 \pm 106.7$ & 0.04 \\
\hline The amount of bleeding until a postoperative drain falls out $(\mathrm{mL})$ & $779.0 \pm 257.3$ & $653.5 \pm 362.9$ & 0.55 \\
\hline
\end{tabular}

PLF, posterior lumbar fusion; PLIF, posterior lumbar interbody fusion.

The improvement rates according to Hirabayashi's method of the PLF group, 1, 3, 6 months, 1 year postoperatively, and at the final follow-up were $61.0 \% \pm 29.6 \%$, $59.5 \% \pm 23.7 \%, 64.4 \% \pm 19.7 \%, 73.5 \% \pm 17.2 \%$, and $70.4 \% \pm 19.1 \%$; those of the PLIF group were $64.2 \% \pm 12.6 \%$, $67.1 \% \pm 14.1 \%, 53.0 \% \pm 35.3 \%, 60.9 \% \pm 42.8 \%$, and $68.4 \% \pm 33.4 \%$, respectively. There was no significant difference between the two groups (Fig. 2).

Results of each parameter in the PLF group and the PLIF group were as follows: hospital stay of $24.5 \pm 8.7$ days and $27.4 \pm 12.2$ days $(p=0.52)$; surgical time of $196.5 \pm 9.5$ minutes and $108.6 \pm 19.5 \mathrm{~min}(p<0.05)$; intraoperative blood loss of $325.5 \pm 191.6 \mathrm{~mL}$ and $177.3 \pm 106.7 \mathrm{~mL}$ $(p<0.05)$; and postoperative blood loss until drainage removal of $779.0 \pm 257.3 \mathrm{~mL}$ and $653.5 \pm 362.9 \mathrm{~mL}(p=0.38)$. Surgical time and intraoperative blood loss were significantly $(p<0.05)$ decreased in the PLIF group compared to those in the PLF group (Table 2).

The following complications occurred. In the PLF group, there were four major complications, including two permanent L5 injuries, one permanent unilateral blindness, and one transient dermatomal pain that was resolved after one month. In the PLIF group, there were 11 major complications: three deep wound infections, two permanent leg pain, two transient leg pain, one permanent drop foot, one transient drop foot, one deep vein thrombosis, and one pulmonary embolism.

\section{Radiographic results}

The mean slip angle in the PLF group decreased from $2.9^{\circ} \pm 7.2^{\circ}$ preoperatively to $-0.5^{\circ} \pm 8.9^{\circ}$ at the final followup. In the PLIF group, it was increased from $6.9^{\circ} \pm 6.2^{\circ}$ preoperatively to $8.9^{\circ} \pm 5.7^{\circ}$ at the final follow-up. This value was significantly $(p<0.05)$ different between the two groups at 3 months postoperatively (Fig. 3A).
Preoperative translation $(\mathrm{mm})$ in the PLF and PLIF groups were $7.2 \pm 3.9 \mathrm{~mm}$ and $5.7 \pm 3.0 \mathrm{~mm}$, respectively $(p=0.33)$. At 1 week postoperatively, the values were $6.4 \pm 4.1 \mathrm{~mm}$ and $1.8 \pm 2.6 \mathrm{~mm}$, respectively $(p=0.005)$. From that time point, the correction of translation was in the PLIF group through the final follow-up was significantly better than that in the PLF group (Fig. 3B). The preoperative slip ratios of the PLF and PLIF groups were $24 \% \pm 11 \%$ and $16 \% \pm 11 \%$, respectively $(p=0.09)$. At 1 week postoperatively, the values were $19 \% \pm 12 \%$ and $4.8 \pm 7.1 \%$, respectively $(p=0.004)$. PLIF resulted in significantly $(p<0.05)$ better correction from this time point through the final follow-up (Fig. 3C). Preoperative vertebral disc heights $(\mathrm{h} / \mathrm{H})$ of the PLF and PLIF groups were $36 \% \pm 15 \%$ and $30 \% \pm 9.8 \%$, respectively $(p=0.35)$. At 1 week postoperatively after surgery, the values were $27 \% \pm 13 \%$ and $47 \% \pm 8.1 \%$, respectively $(p=<0.001)$. The PLIF group showed significantly $(p<0.05)$ higher vertebral disc height from this time point through the final follow-up (Fig. 3D). Preoperative lumbar lordosis (L1-S1) of the PLF and PLIF groups were $38^{\circ} \pm 15^{\circ}$ and $38^{\circ} \pm 12^{\circ}$, respectively $(p=0.70)$. At the final follow-up, the values were $27^{\circ} \pm 24^{\circ}$ and $37^{\circ} \pm 7^{\circ}$, respectively $(p=0.12)$. There was no significant difference between the two groups (Fig. 4).

The preoperative slip angles on maximum flexion and extension in the PLF and PLIF groups were $8.0^{\circ} \pm 5.3^{\circ}$ and $12.0^{\circ} \pm 5.1^{\circ}$, respectively $(p=0.04)$. The PLIF group was significantly unstable. However, at 3 months postoperatively, the values were $5.7^{\circ} \pm 3.4^{\circ}$ and $1.9^{\circ} \pm 2.5^{\circ}$, respectively $(p=0.02)$. At 6 months postoperatively, the values were $3.9^{\circ} \pm 2.3^{\circ}$ and $1.5^{\circ} \pm 1.7^{\circ}$, respectively $(p=0.02)$. Thus, the slip angle of the PLIF group was more limited. However, at the final follow-up, the slip angles of the PLF and PLIF groups were $3.3^{\circ} \pm 1.6^{\circ}$ and $3.1^{\circ} \pm 2.4^{\circ}$, respectively $(p=0.8)$ (Fig. 5A). Preoperative translation on maximum flexion and extension in the PLF and PLIF groups were 2.3 \pm 1.4 

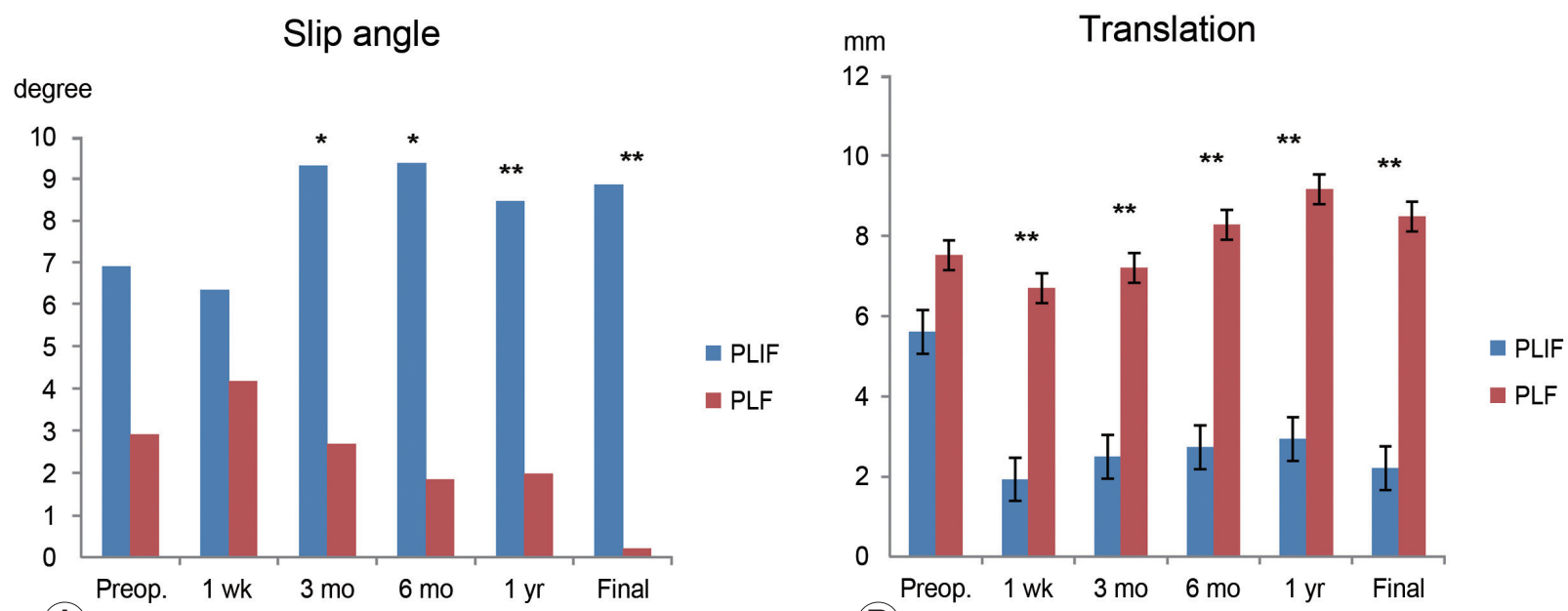
(A)

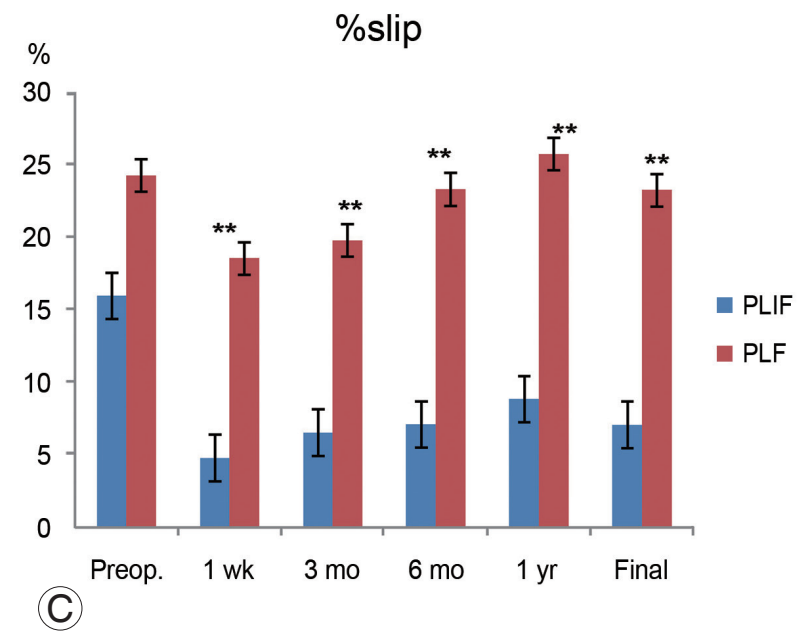

(B)

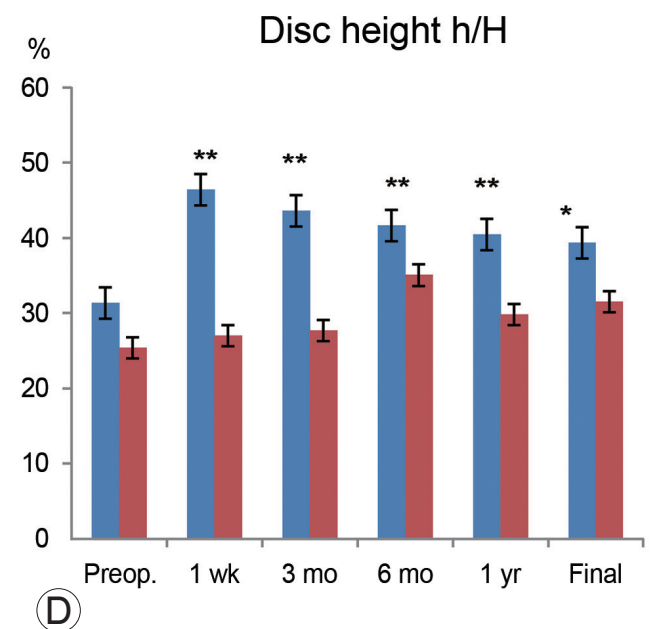

Fig. 3. (A-D) Radiologic data of the operated segment. Postoperative correction was better and the vertebral disc height was higher in the PLIF group, although the preoperative slip angle and translation were not significantly different between the two groups. Preop., preoperative; PLIF, posterior lumbar interbody fusion; PLF, posterior lumbar fusion; $h$, disc height; $H$, posterior wall height of the proximal vertebral body. ${ }^{*} p<0.05,{ }^{* *} p<0.01$.

$\mathrm{mm}$ and $3.9 \pm 2.5 \mathrm{~mm}$, respectively $(p=0.1)$. At 3 months postoperatively, the values were $2.2 \pm 1.3 \mathrm{~mm}$ and $0.9 \pm 1.0$ $\mathrm{mm}$, respectively $(p=0.04)$. Thus, the PLIF group showed significantly limited translation in motion. However, there was no significant difference at 6 months postoperatively or at the final follow-up (PLF and PLIF group: 2.0 \pm 1.8 $\mathrm{mm}$ and $1.6 \pm 1.7 \mathrm{~mm}$, respectively, $p=0.5$ ) (Fig. $5 \mathrm{~B}$ ). The fusion rates of the PLF and PLIF groups were $72.3 \%$ and $89.5 \%$, respectively ( $p>0.05$ ) (Fig. 6).

\section{Discussion}

The theoretical advantages of PLIF include anterior column support, indirect foraminal decompression, restora- tion of lordosis, and maintenance of intervertebral disc height [17]. Anterior column augmentation in the PLIF procedure for degenerative spondylolisthesis, which employs autologous iliac bone graft in addition to pedicular screw fixation and PLF, is likely to achieve better fusion rate and clinical results [18]. Crawford et al. [19] reproduced grade I lumbar spondylolisthesis using cadaveric specimens and studied the biomechanics of various instrumentation combinations, including cages with or without intersomatic spacers, pedicle screws alone, and pedicle screws with cages. Pedicle screws with cages presented better biomechanics in flexion, lateral extension, axial rotation, and shear force. Specimens with cages showed instability and fatigue. They recommended the 
use of screw systems and cages in grade I lumbar spondylolisthesis patients because greater stability might allow good fusion around the cages.

Suk et al. [13] recommended the PLIF procedure because PLF was associated with more cases of pseudoarthrosis while PLIF has preferable characteristics such as anterior column support, maintenance of reduction, and a better fusion rate. Kim et al. [20] compared PLF, PLIF, and the combination the two and found that PLIF had better surgical time, blood loss, and donor site pain, al-

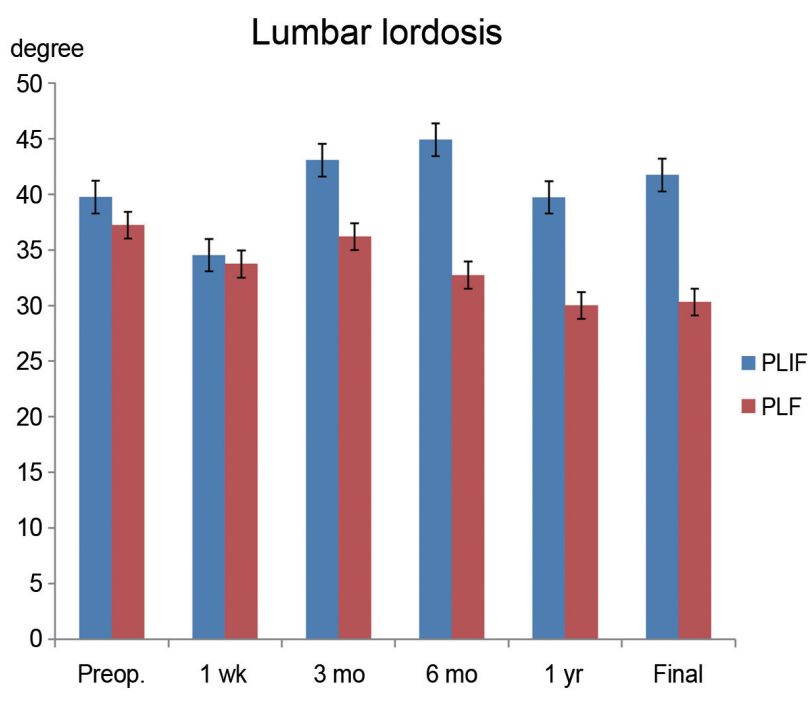

Fig. 4. Radiologic data for the sagittal alignment. Lumbar lordosis was not significantly different between the two groups. Preop., preoperative; PLIF, posterior lumbar interbody fusion; PLF, posterior lumbar fusion. though the clinical results of the three were similar. In our study, the PLIF group showed significantly shorter surgical time. However, as PLIFs were performed by two experienced doctors in one facility while PLFs were mainly performed in a university hospital by two senior spine surgeons and 4 fellows, learning curve might have influenced the result. Musluman et al. [21] reported that PLIF could achieve better sagittal balance and that the back pain visual analogue scale (VAS) scores were improved from the early postoperative period to the final followup. However, some authors have reported that the clinical results of PLIF are not superior than PLF in the treatment of low-grade spondylolisthesis [20-23], although PLIF is expected to achieve better maintenance of correction and

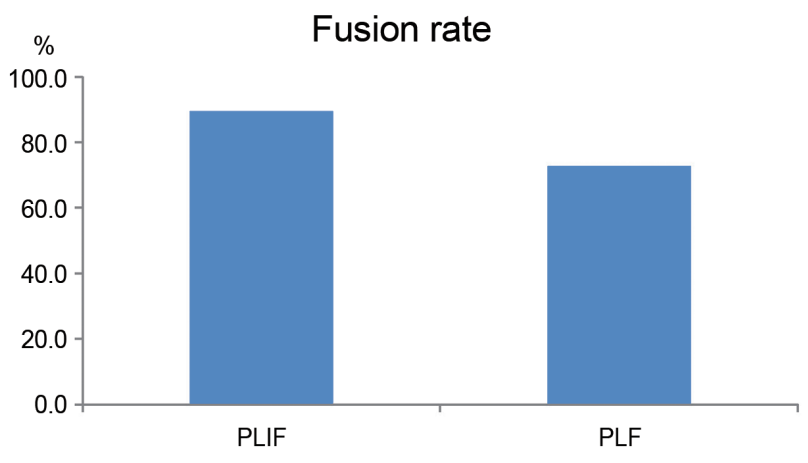

Fig. 6. Fusion rate. The bony union rate at the final follow-up was not significantly different between the two groups. Preop., preoperative; PLIF, posterior lumbar interbody fusion; PLF, posterior lumbar fusion.

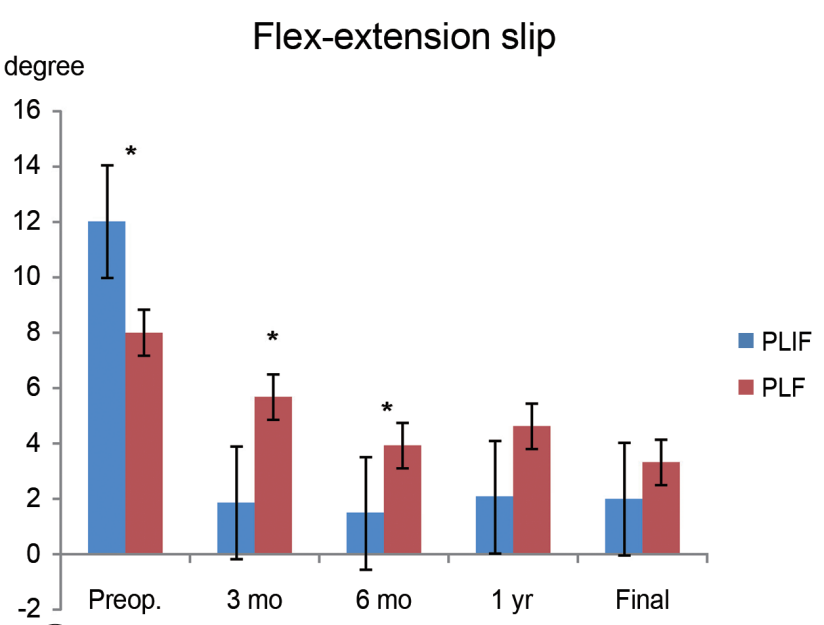

(A)

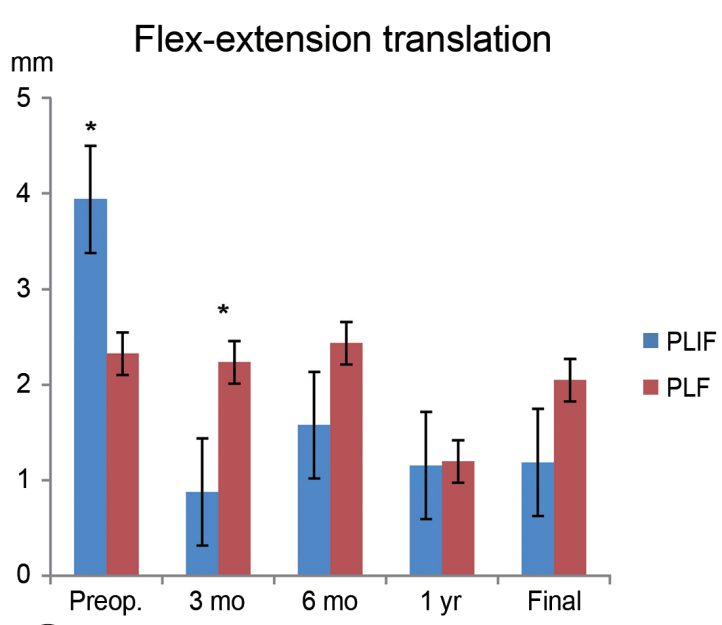

(B)

Fig. 5. (A, B) Radiologic data on maximum flexion and extension. Preoperative instability on maximum flexion and extension was stronger in the PLIF group. However, the PLF group showed instability at 3 and 6 months postoperatively compared to the PLIF group. The instability in the maximum flexion and extension disappeared in both groups. Preop., preoperative; PLIF, posterior lumbar interbody fusion; PLF, posterior lumbar fusion. ${ }^{*} p<0.05,{ }^{* *} p<0.01$. 
bony union.

Most previous reports compared cases of spondylolysis/ spondylolisthesis or multi-vertebral level. Few studies compared the results at a single vertebrae level. Ha et al. [18] compared PLF and PLIF for degenerative lumbar spondylolisthesis at single level at L4/5 in regard to clinical outcomes and radiographic parameters. They classified subjects into a stable group and an unstable group using a degree of slip threshold of $4 \mathrm{~mm}$ and a slip angle of $10^{\circ}$. The clinical outcomes in the stable group for PLF and PLIF were similar to each other. However, PLIF resulted in better clinical outcomes in the unstable group. They also reported that PLF in unstable group without anterior support showed higher possibility of sagittal imbalance caused by disc space re-narrowing, progression of displacement, loss of reduction, or non-union. However, in their study, the vertebral disc height increased in both stable and unstable groups, while the degree of slip, lordosis angle, and sacral tilt was not affected by insertion of the cage. In the present study, PLIF and PLF for unstable degenerative spondylolisthesis were compared. We found that their clinical results were not significantly different. Subjects with greater instability were defined by degree of slip $\geq 4 \mathrm{~mm}$, slip angle $\geq 10^{\circ}$, posterior opening on maximum flexion $\geq 5^{\circ}$, and Mayerding grade $\geq$ II. However, some subjects with a degree of slip $<4 \mathrm{~mm}$ and slip angle $<10^{\circ}$ were also included in this study. In this study, PLIF showed better results in the correction of slip, maintenance of vertebral disc height, and fusion rate. However, lumbar lordosis in the PLIF group was not better than that in the PLF group, which might be due to the lack of difference in clinical outcomes between the two groups.

Some authors consider PLIF procedure difficult due to increased bleeding, prolonged operation time, and more extensive dissection [15,24-27]. Complications associated with the PLIF procedure include permanent neurological deficit $(0.4 \%-1.7 \%)$, cerebrospinal fluid leakage $(0.4 \%-$ $0.5 \%)$, radicular pain $(1.1 \%-2.5 \%)$, posterior cage dislocation $(0.8 \%-0.9 \%)$, and deep wound infection $(0.6 \%-5 \%)$ [1,18,28-30]. Musluman et al. [21] have reported that in PLIF, complications due to nerve root retraction are found in only one patient (4\%). Those complications are not permanent. This low rate of neurological deficits may be attributed to the insertion of PLIF cages in a manner similar to the transforaminal lumbar interbody fusion approach after bilateral facetectomy and laminectomy, which can minimize nerve root retraction. The average bleeding volume in the PLF group was $270 \mathrm{~mL}$, which was greater than that in the PLIF group. In the PLF group, there were 4 major complications (2 permanent L5 injuries, 1 patient with permanent unilateral blindness, and 1 patient with transient dermatomal pain, which resolved after 1 month). In the PLIF group, there were 11 major complications (3 deep wound infections, 2 patients with permanent leg pain, 2 with transient leg pain, 1 patient with permanent and 1 with transient drop foot, 1 patient with a deep vein thrombosis, and 1 patient with a pulmonary embolism). The higher number of complications in the PLIF group might have resulted from a more invasive nature of total facetectomy or discectomy. In contrast, PLF employs in situ fixation. Although the PLIF group showed a better slip correction rate, root injury might have occurred during correction. The limitations of this study include the following. First, multiple surgeons in the two facilities performed the surgeries. Second, the case number used in this study was small. Third, the follow-up rate in the PLF group was low. Fourth, clinical evaluation in this study was not performed by patients-oriented measure.

\section{Conclusions}

PLF was compared to PLIF for patients with unstable L4 spondylolisthesis. Clinical outcomes by JOA scores were not significantly different between the two groups. Postfixation correction was better in the PLIF group. However, no significant difference was observed for lumbar lordosis between the two groups.

\section{Conflict of Interest}

No potential conflict of interest relevant to this article was reported.

\section{References}

1. Feffer HL, Wiesel SW, Cuckler JM, Rothman RH. Degenerative spondylolisthesis: to fuse or not to fuse. Spine (Phila Pa 1976) 1985;10:287-9.

2. Herkowitz HN, Kurz LT. Degenerative lumbar spondylolisthesis with spinal stenosis: a prospective study comparing decompression with decompression and intertransverse process arthrodesis. J Bone Joint Surg Am 1991;73:802-8.

3. Booth KC, Bridwell KH, Eisenberg BA, Baldus CR, 
Lenke LG. Minimum 5-year results of degenerative spondylolisthesis treated with decompression and instrumented posterior fusion. Spine (Phila Pa 1976) 1999;24:1721-7.

4. Bridwell KH, Sedgewick TA, O’Brien MF, Lenke LG, Baldus $\mathrm{C}$. The role of fusion and instrumentation in the treatment of degenerative spondylolisthesis with spinal stenosis. J Spinal Disord 1993;6:461-72.

5. Sudo H, Oda I, Abumi K, Ito M, Kotani Y, Minami A. Biomechanical study on the effect of five different lumbar reconstruction techniques on adjacent-level intradiscal pressure and lamina strain. J Neurosurg Spine 2006;5:150-5.

6. Barrick WT, Schofferman JA, Reynolds JB, et al. Anterior lumbar fusion improves discogenic pain at levels of prior posterolateral fusion. Spine (Phila Pa 1976) 2000;25:853-7.

7. Lee CK, Vessa P, Lee JK. Chronic disabling low back pain syndrome caused by internal disc derangements. The results of disc excision and posterior lumbar interbody fusion. Spine (Phila Pa 1976) 1995;20:35661.

8. Brantigan JW, Neidre A. Achievement of normal sagittal plane alignment using a wedged carbon fiber reinforced polymer fusion cage in treatment of spondylolisthesis. Spine J 2003;3:186-96.

9. Arai Y, Takahashi M, Kurosawa H, Shitoto K. Comparative study of iliac bone graft and carbon cage with local bone graft in posterior lumbar interbody fusion. J Orthop Surg (Hong Kong) 2002;10:1-7.

10. McAfee PC, DeVine JG, Chaput CD, et al. The indications for interbody fusion cages in the treatment of spondylolisthesis: analysis of 120 cases. Spine (Phila Pa 1976) 2005;30(6 Suppl):S60-5.

11. Zhao J, Wang X, Hou T, He S. One versus two BAK fusion cages in posterior lumbar interbody fusion to L4-L5 degenerative spondylolisthesis: a randomized, controlled prospective study in 25 patients with minimum two-year follow-up. Spine (Phila Pa 1976) 2002;27:2753-7.

12. Ito Z, Matsuyama $Y$, Sakai Y, et al. Bone union rate with autologous iliac bone versus local bone graft in posterior lumbar interbody fusion. Spine (Phila Pa 1976) 2010;35:E1101-5.

13. Suk SI, Lee CK, Kim WJ, Lee JH, Cho KJ, Kim HG. Adding posterior lumbar interbody fusion to pedicle screw fixation and posterolateral fusion after decom- pression in spondylolytic spondylolisthesis. Spine (Phila Pa 1976) 1997;22:210-9.

14. Hirabayashi K, Miyakawa J, Satomi K, Maruyama T, Wakano K. Operative results and postoperative progression of ossification among patients with ossification of cervical posterior longitudinal ligament. Spine (Phila Pa 1976) 1981;6:354-64.

15. Madan S, Boeree NR. Outcome of posterior lumbar interbody fusion versus posterolateral fusion for spondylolytic spondylolisthesis. Spine (Phila $\mathrm{Pa}$ 1976) 2002;27:1536-42.

16. Freeman BJ, Licina P, Mehdian SH. Posterior lumbar interbody fusion combined with instrumented postero-lateral fusion: 5-year results in 60 patients. Eur Spine J 2000;9:42-6.

17. van Dijk M, Smit TH, Sugihara S, Burger EH, Wuisman PI. The effect of cage stiffness on the rate of lumbar interbody fusion: an in vivo model using poly(1-lactic Acid) and titanium cages. Spine (Phila Pa 1976) 2002;27:682-8.

18. Ha KY, Na KH, Shin JH, Kim KW. Comparison of posterolateral fusion with and without additional posterior lumbar interbody fusion for degenerative lumbar spondylolisthesis. J Spinal Disord Tech 2008; 21:229-34.

19. Crawford NR, Cagli S, Sonntag VK, Dickman CA. Biomechanics of grade I degenerative lumbar spondylolisthesis. Part 1: in vitro model. J Neurosurg 2001;94:45-50.

20. Kim KT, Lee SH, Lee YH, Bae SC, Suk KS. Clinical outcomes of 3 fusion methods through the posterior approach in the lumbar spine. Spine (Phila Pa 1976) 2006;31:1351-7.

21. Musluman AM, Yilmaz A, Cansever T, et al. Posterior lumbar interbody fusion versus posterolateral fusion with instrumentation in the treatment of lowgrade isthmic spondylolisthesis: midterm clinical outcomes. J Neurosurg Spine 2011;14:488-96.

22. Dantas FL, Prandini MN, Ferreira MA. Comparison between posterior lumbar fusion with pedicle screws and posterior lumbar interbody fusion with pedicle screws in adult spondylolisthesis. Arq Neuropsiquiatr 2007;65:764-70.

23. Cheng L, Nie L, Zhang L. Posterior lumbar interbody fusion versus posterolateral fusion in spondylolisthesis: a prospective controlled study in the Han nationality. Int Orthop 2009;33:1043-7. 
24. Fraser RD. Interbody, posterior, and combined lumbar fusions. Spine (Phila Pa 1976) 1995;(24 Suppl):167S-177S.

25. Inamdar DN, Alagappan M, Shyam L, Devadoss S, Devadoss A. Posterior lumbar interbody fusion versus intertransverse fusion in the treatment of lumbar spondylolisthesis. J Orthop Surg (Hong Kong) 2006; 14:21-6.

26. Rompe JD, Eysel P, Hopf C. Clinical efficacy of pedicle instrumentation and posterolateral fusion in the symptomatic degenerative lumbar spine. Eur Spine J 1995;4:231-7.

27. Thomsen K, Christensen FB, Eiskjaer SP, Hansen ES, Fruensgaard S, Bunger CE. 1997 Volvo Award winner in clinical studies. The effect of pedicle screw instrumentation on functional outcome and fusion rates in posterolateral lumbar spinal fusion: a prospective, randomized clinical study. Spine (Phila Pa 1976) 1997;22:2813-22.

28. Davne SH, Myers DL. Complications of lumbar spinal fusion with transpedicular instrumentation. Spine (Phila Pa 1976) 1992;17:S184-9.

29. Lin PM. Posterior lumbar interbody fusion technique: complications and pitfalls. Clin Orthop Relat Res 1985;(193):90-102.

30. Weatherley CR, Prickett CF, O’Brien JP. Discogenic pain persisting despite solid posterior fusion. J Bone Joint Surg Br 1986;68:142-3. 